\title{
Radiographical and Histological Evaluation of Canine Decidual Reaction Induced by Intralumenal Injection of Bouillon Solution Mixed with or without Barium Sulfate
}

\author{
Koichi NOMURA \\ Laboratory of Surgery, Department of Veterinary Science, College of Agriculture, University of Osaka Prefecture, 1-1 Gakuen-cho, \\ Sakai, Osaka 593, Japan
}

(Received 2 May 1995/Accepted 25 September 1995)

ABSTRACT. Radiographical examination of the canine uterus intralumenally injected with barium solution revealed that barium was
gradually condensed, segmented and distributed equally along the lumen like embryos in early pregnant uterus. Histological examination
of the uterus intralumenally injected with bouillon solution alone showed a remarkable fish net- or tree branch-like growth of superficial
glands towards the uterine lumen. The uterus injected with barium in bouillon solution induced two different histological changes: One
consisted of cystic glandular hyperplasia in the whole endometrium, which was a so-called Swiss cheese endometrium, and the other was
composed of a fish net- or tree branch-like growth in the superficial layer accompanied with cystic glandular hyperplasia in the basal
layer. The latter represented endometrial differentiation into spongy layer, supraglandular layer and basal glandular layer, being similar to
that during normal early placentation. These findings suggest that clotted barium in bouillon solution has stimulating effects on the uterus
similar to those of embryos. - KEY wORDS: barium solution, bouillon solution, deciduoma.

It is generally accepted that the dog is the deciduata and that the decidual reaction in a pregnant dog is characterized by remarkable proliferation of the uterine glands, not by proliferation of the stromal cells as seen in the rodentia [1$3,5]$. In the deciduata, a decidua like mass which can be induced by artificial stimuli in the diestrous endometrium is called "deciduoma" [4], used as a model for studying the mechanism of implantation [14]. In the dog, however, histological structure of the deciduoma has not been clarified. Recently we reported that canine deciduoma could be induced by various methods used in the rodentia and that the histological findings observed were characterized by remarkable proliferation and dilatation of uterine glands [11]. However, the changes were limited either in functional layer or basal layer apart from those observed in the normal early placentation $[10,12,13]$.

We previously could find the facts radiographically that a barium solution formed some masses in the diestrous uterine lumen and that they were segmentally distributed like as embryos in the early pregnancy [9]. From these findings, we obtained an idea to inject barium solution as a favarable stimulus for induction of the deciduoma that would be a suitable model for implantation.

This study deals with radiographical and histological evaluation of canine decidual reactions induced by intraluminal injection of a bouillon solution with or without barium sulfate.

Fifteen adult mongrel bitches from random sources, weighing 8 to $12 \mathrm{~kg}$, were used. In anestrus, each animal received laparotomy under general anesthesia with xylazine hydrochloride and ketamine hydrochloride. Both oviducts were ligated with silk suture to prevent pregnancy. Each bitch was monitored daily for estrous cycle by external genital signs and vaginal cytology. During estrus, each bitch was allowed to copulate with a male dog. The first

\footnotetext{
*Trypto-Soya Broth "Nissui": produced by Nissui pharmaceutical company in Tokyo, Japan.
}

day of diestrus (day 1) was determined by the refusal behavior for male and vaginal cytology [7].

The bitches were divided into three groups consisting of five bitches each. On day 12, the bitches were laparotomized again and received the following treatments under general anesthesia. [Group 1] $0.5 \mathrm{ml}$ of bouillon solution (TryptoSoya Broth*) was injected intralumenally into the each uterine horn using a sterilized syringe with a $25 \mathrm{G} \times 5 / 8^{\prime \prime}$ gauge needle. [Group 2] $0.5 \mathrm{~m} l$ of the solution mixed barium sulfate with sterilized bouillon (1:1) (barium solution) was injected intralumenally into the each uterine horn by the same method as was used in group 1 . Uterine roentgenograms were serially taken to monitor how the barium is distributed along the lumen. [Group 3] $0.5 \mathrm{~m} l$ of sterilized physiological saline was injected intralumenally into the each uterine horn as a control.

On day 24 , all bitches were ovariohysterectomized by a usual operative procedure under general anesthesia. The removed ovaries and uteri were observed grossly and then fixed in $10 \%$ neutral buffered formalin. Histological sections from them were made routinely and stained with hematoxylin and eosin (H E) and, if necessary, periodic acid Schiff (PAS) or Azan-Mallory Heidenhain.

All surgeries were performed by a standard aseptic technique and daily injection of an antibiotic (aminophenyl acetamido penicillanic acid $10 \mathrm{mg} / \mathrm{kg} /$ day) was given to all bitches until the end of the experiment.

Radiographical findings: This examination was available only in group 2 dogs. At ten minutes after the injection, the outline of uterine lumen was a long waving line (Fig. 1). At 90 min after the injection, the barium was gradually condensed and divided into some isolated masses (Fig. 2). The masses further altered their shape to a comma-like pattern. At 6 days after the injection, the segmented contents were equally distributed along the lumen just like the embryos seen in the early pregnant uterus (Fig. 3).

Gross findings: In group 1, the endometrium was slightly hypertrophic and edematous and had no wrinkles. In some 
cases, watery contents in the lumen and thin uterine walls were seen. In group 2, bends and twists were formed on the serosa of the uterus, and rough annular bands were also frequently seen on the serosa. The uterine wall was congested and edematous. The endometrium was remarkably hyperplastic. By the folds corresponding to the bands, the uterine lumen was divided into small compartments in which barium was seen to be clotted. The clotted barium masses showed a variety of shapes (Fig. 4). In group 3, gross findings of the uterus were similar to those in the normal middle or late diestrous stage.

Histological findings: In the uterus of group 1, bouillon was still remaining in the lumen. Epithelial and glandular proliferation of the superficial endometrial layer was prominent, forming a fish-net-like appearance. Proliferation of stromal cells was not clear. In the uterus of group 2, the superficial endometrium which was facing to the fluid fraction in the content was remarkably developed toward the lumen to form a fish net or tree branch-like structure. Some crypts of the superficial glands dilated to form a primitive lacunae (Fig. 5). Under this layer, fibrous connective tissue was formed. This layer separated the endometrium into two parts: the superficial functional layer and basal glandular layer. Barium particles were observed

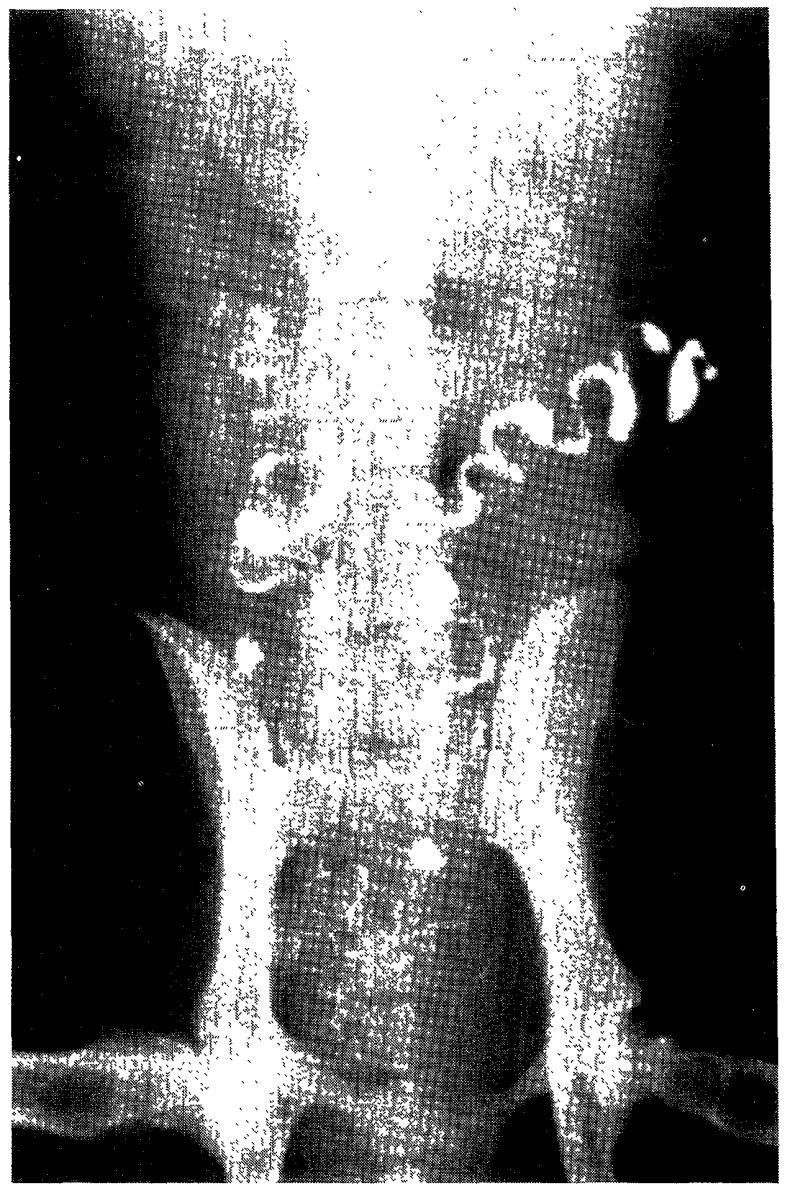

Fig. 1. Roentgenogram of the uterus taken $10 \mathrm{~min}$ after injection of the barium solution. Contents in the uterine lumen appear to be irregular in shape. in some glandular lumina in the basal layer. On the other hand, the endometrium directly touching the condensed barium showed a marked cystic glandular hyperplasia of the basal layer (Fig. 6). It could be so-called "Swiss cheese endometrium". The uterus of group 3 showed almost same findings seen in the normal diestrous uterus, showing a slight hypertrophy of superficial glands and fibrous hyperplasia of the stroma. The histological findings of the ovary showed no difference among all groups. Each ovary had matured or nearly matured corpora lutea which can be seen in the normal diestrous stage.

In the uterus injected with barium, "compartments" were formed by dividing the lumen into small sections, resembling the implantational chambers seen in the normal early pregnancy. A barium mass was situated in the center of each compartment as if it were an embryo in the placenta. This fact suggests that when the diestrous uterus recognized contents as an acceptable item, even though they were foreign bodies, the uterus could distribute them as if they were embryos in the implantational phase.

In the rodentia, the placenta-like mass induced by an artificial stimulation in the diestrous uterus is termed "deciduoma" [4]. It mainly consists of proliferated stromal cells reflecting histological characteristics of their normal

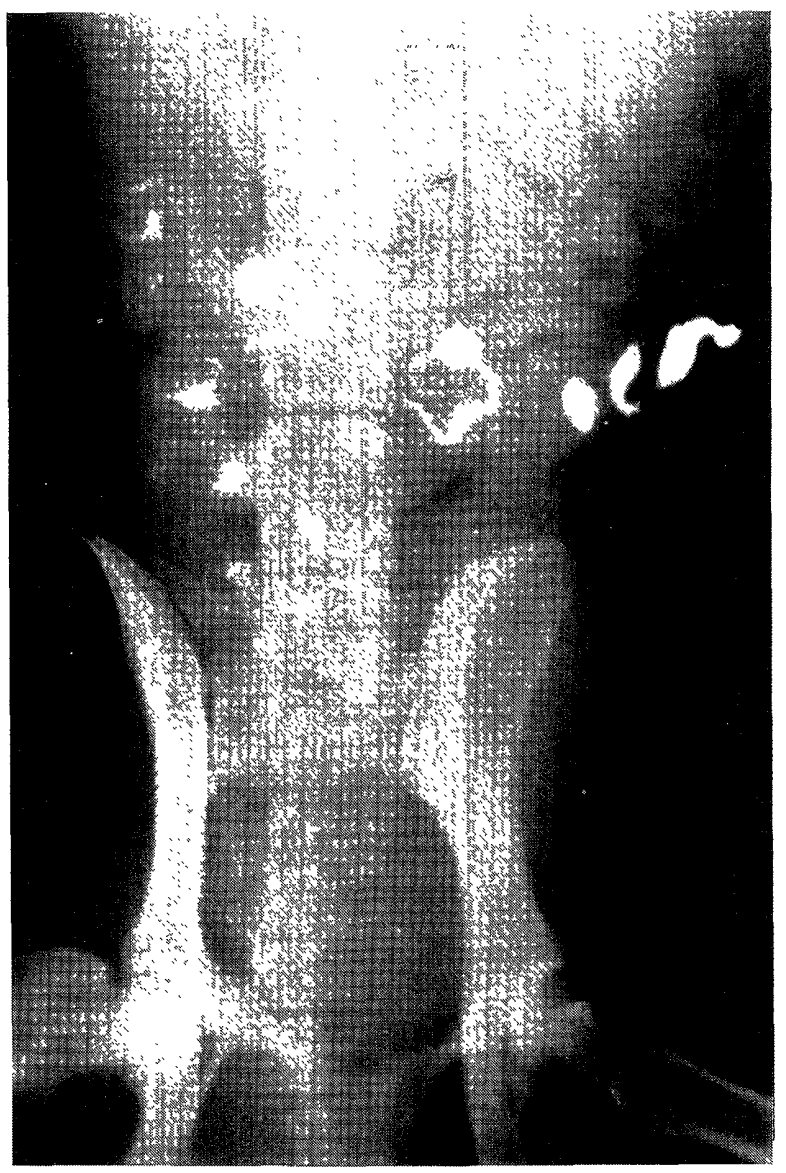

Fig. 2. Roentgenogram of the same uterus in Fig. 1 taken $90 \mathrm{~min}$ after the injection. Uterine contents altered their shape from segmented to comma-like. 


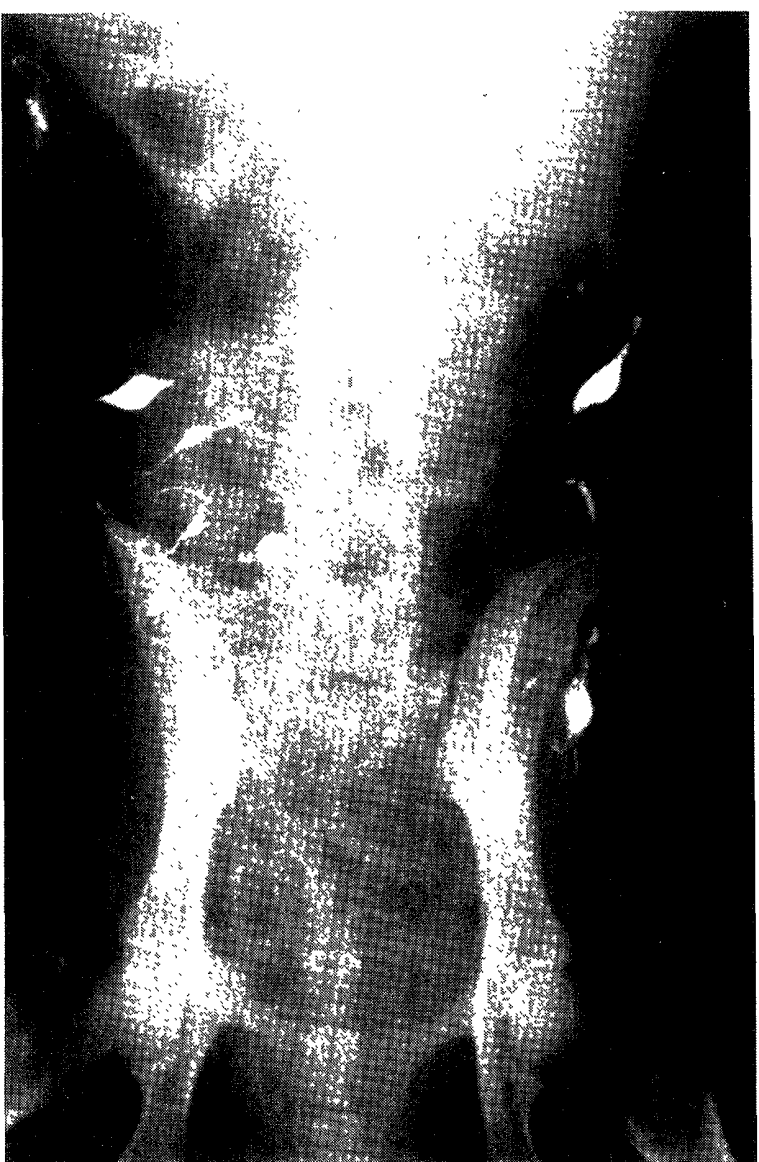

Fig. 3. Roentgenogram taken 6 days after the injection. The barium masses in the uterus are condensed, individually separated, and finally distributed as if they were fertilized eggs in pregnancy.

early placentation. On the other hand, normal dog placenta and canine deciduoma are composed of proliferated uterine glands $[1-3,11]$. Thus, in the deciduata animals, the histological structure of the deciduoma is different among species. During the normal process of placentation in dogs, different histological stages are recognised depending on the development of uterine glands [6]. Especially in the early stage of implantational process, drastic histological changes can be seen in the transformation of the uterine glands, i.e., the glands in the superficial layer develope to form of crypts and lacnae, and finally a spongy layer [3]. In our previous reports, for examples, liquid stimuli such as olive oil which remains in the lumen for a relatively long period permitted to induce remarkable development of the superficial glands toward the uterine lumen [13]. Flexible but solid stimuli such as silk suture induced the growth of the glands toward the muscle layer because this stimulus might give various strength of pressure to the surface of the endometrium and disturb the tissue to develop intraluminally [12]. Traumatic stimuli such as scratching with wire or uterine inoculation of $E$. coli broth culture induced cystic glandular hyperplasia in the basal layer, which was the socalled "Swiss cheese endometrium". It was supposed that such cystic glandular hyperplasia was caused by the traumatic occlusion of the openings of glands $[8,10]$. Thus various patterns of histological changes depended on the

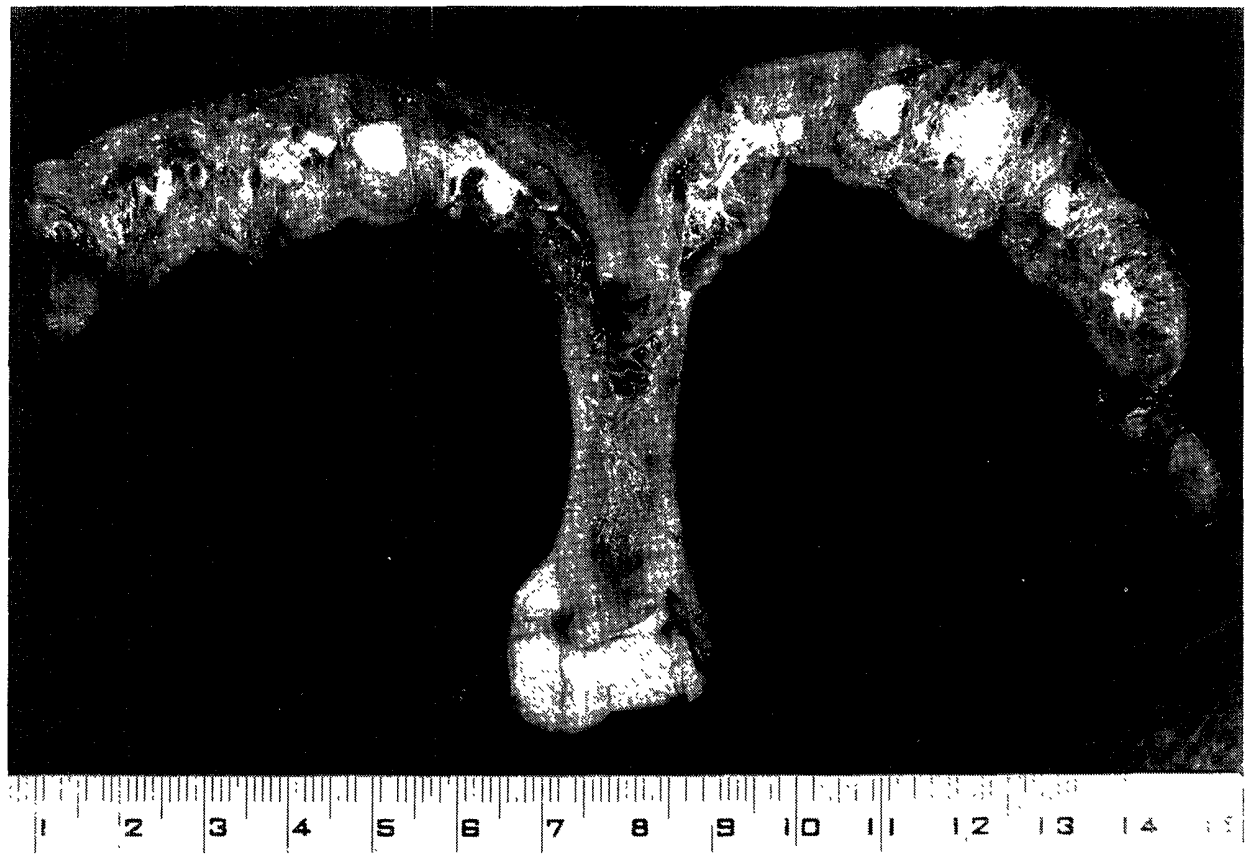

Fig. 4. Hypertrophic mucosal surface of the uterus which was intraluminally injected with a barium solution (group 2). Uterine lumen is divided into some compartments by the annular folds. in which various volumes of the clotted barium remain . 


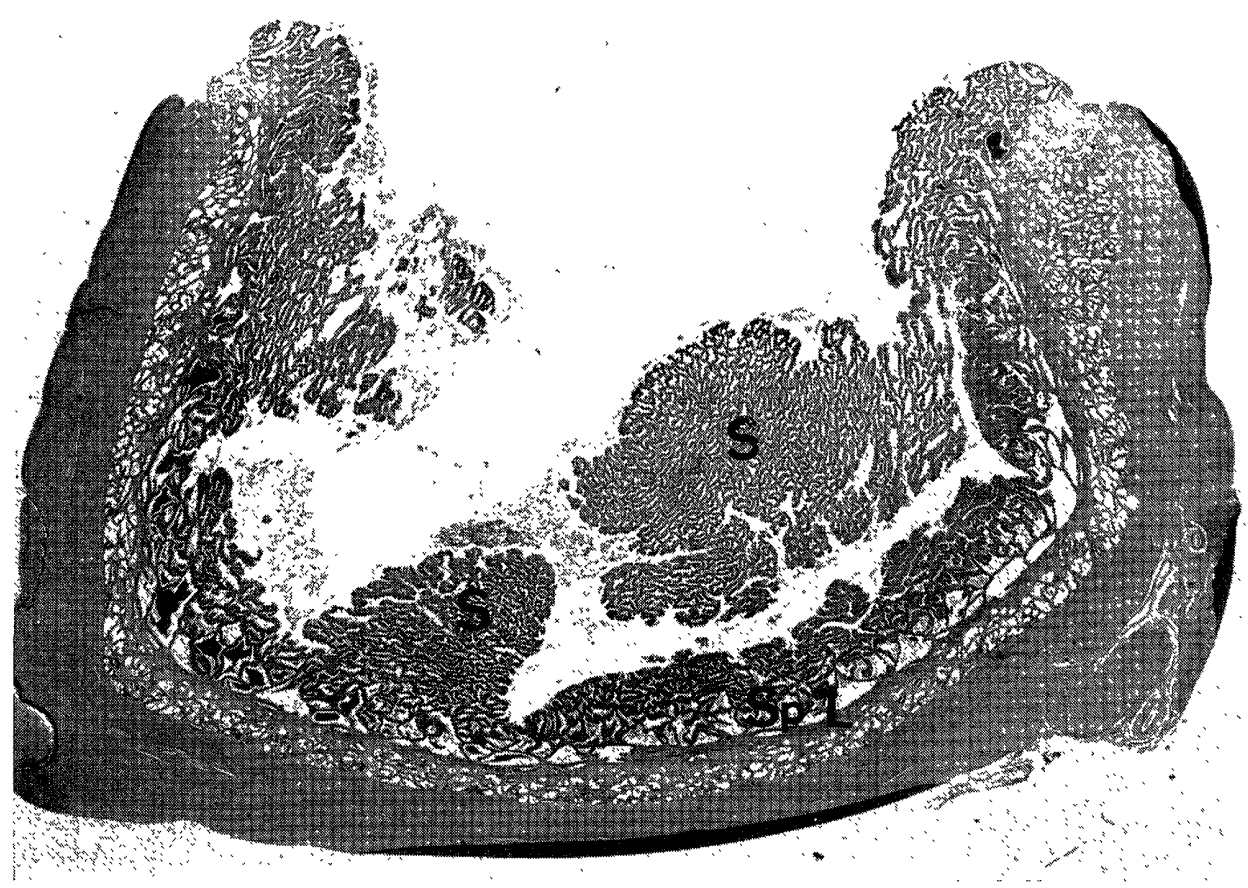

Fig. 5. Histological picture from the uterus injected with barium solution (group 2). The endometrium does not directly touch the barium mass but faces the bouillon. The endometrium is growing actively into the lumen and forms the structure which looks like a fish net or tree branches (S). Under this layer, premature spongy layer (Sp. L.) was formed by dilatation of a few glands. HE stain. $\times 15$.

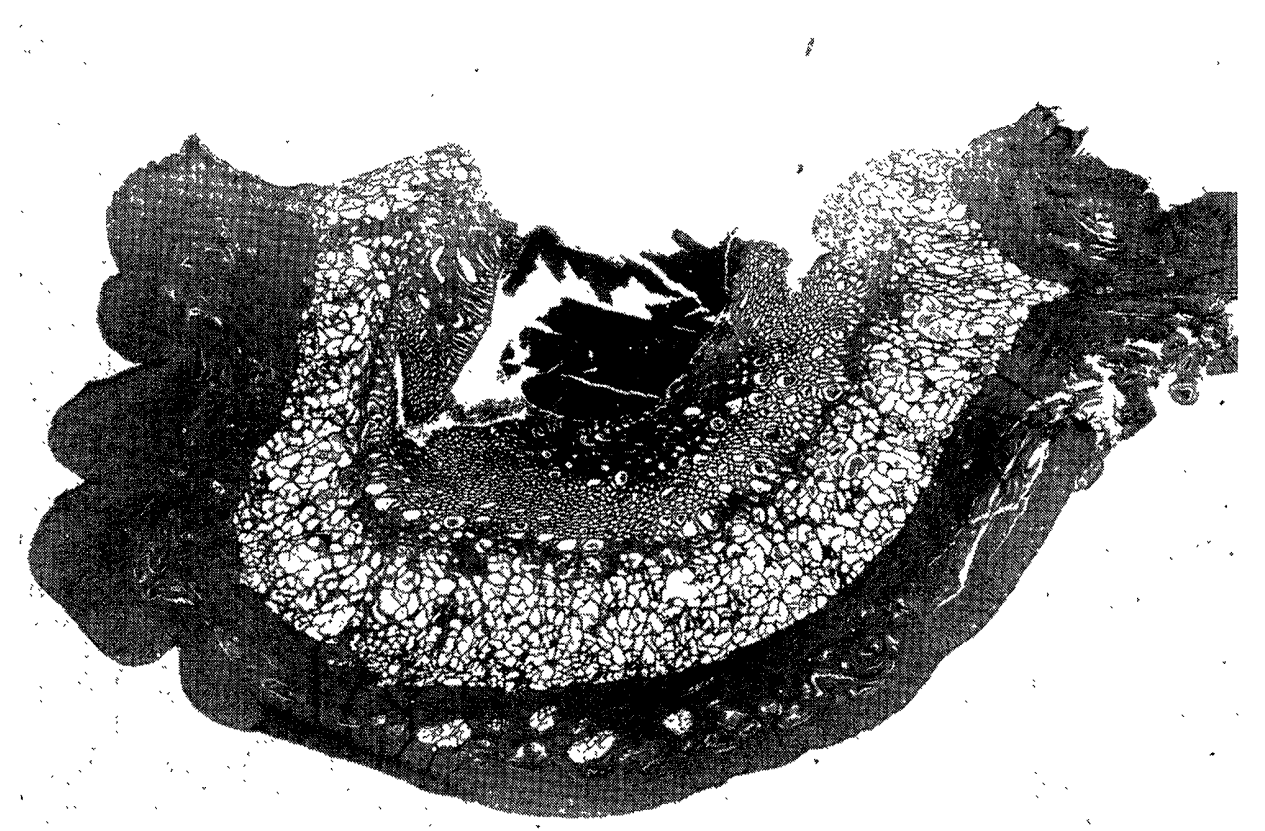

Fig. 6. Another histological picture from the uterus injected with barium solution (group 2). This picture shows the area where the endometrium directly touched the barium mass. Cystic glandular hyperplasia is seen in the whole endometrium. HE stain. $\times 15$.

differences of the timing or natures of the artificial stimuli.

In the present study, we used the bouillon solution with or without barium sulfate as stimulants. Bouillon solution itself induced similar endometrial change like as olive oil [13]. Concentrated barium sulfate in bouillon solution, on the other hand, induced traumatic stimuli by rubbing endometrium directly. The trauma may cause occlusion of the glandular openings and then dilatation of the lumina. The endometrium may gradually become hyperplastic with cystic dilatation of the glands in all the layers. On the 
contrary, some places where the endometrium faced indirectly to the barium mass may allow the superficial tissue to grow toward the lumen at first, showing a fish netor tree branch-like structure. In this way, stimulation by the barium mass floating in the bouillon solution may induce endometrial hyperplasia with fish net-like proliferation in the superficial layer and cystic hyperplasia in the basal layer. This structure seems like normal early placenta.

Therefore, the deciduoma induced by the barium solution would provide the most appropriate histological model for placental development in the early pregnancy.

\section{REFERENCES}

1. Amoroso, E. C. 1952. pp. 138-159. In: Marshall's Physiology of Reproduction, vol. 2, 3rd ed. (Parkes, A. S. ed.), Longmans Green and Co., London, New York, Toronto.

2. Amoroso, E. C. 1952. pp. 211-242. In: Marshall's Physiology of Reproduction, vol. 2, 3rd ed. (Parkes, A. S. ed.), Longmans Green and Co., London, New York, Toronto.
3. Barrau, M. D., Abel, J. H., Torbit, C. A., and Tietz, W. J. 1975. Am. Anat. 143: 115-130.

4. De Feo, V. J. 1967. pp. 191-249. In: Cellular Biology of the Uterus (Wynn, R. M. ed.), Appletone, Century, Crofts.

5. Enders, A. C. 1970. pp. 152-153. In: Reproduction and Breeding Techniques for Laboratory Animals (Hafez, E. S. E. ed.), Lea \& Febiger, Philadelphia.

6. Heinricius, G. 1889. Archiv Mikroskop. Anatomie 33: 419439.

7. Holst, P. A. and Phemister, R. D. 1974. J. Vet. Res. 35: 401406.

8. Nomura, K. 1982. Jpn. J. Vet. Sci. 45: 237-240.

9. Nomura, K., Yosida, K., and Shimada, Y. 1987. J. Jpn. Vet. Med. Assoc. 40: 567-573.

10. Nomura, K., Kawasoe, K., and Shimada, Y. 1990. Jpn. J. Vet. Sci. 52: 979-983.

11. Nomura, K. 1994. J. Vet. Med. Sci. 56: 365--369.

12. Nomura, K. 1995. J. Vet. Med. Sci. 57: 9-16.

13. Nomura, K. 1995. J. Vet. Med. Sci. 57: 71-74.

14. Shelesnyak, M. C. 1957. Recent Prog. Horm. Res. 13: 269322 . 